\title{
The importance of response type to the relationship between temporal order and numerical magnitude
}

\author{
Michael E. R. Nicholls • Megan Lew • \\ Tobias Loetscher • Mark J. Yates
}

Published online: 9 March 2011

(C) Psychonomic Society, Inc. 2011

\begin{abstract}
Time, number, and space may be represented in the brain by a common set of cognitive/neural mechanisms. In support of this conjecture, Schwarz and Eiselt (Journal of Experimental Psychology: Human Perception and Performance, 35, 989-1004, 2009) found that numerically smaller digits were perceived to occur earlier than larger digits, and they concluded that this difference reflected faster processing of smaller numbers. This difference, however, could have been related to a response bias, whereby participants map responses of "which first" onto the "first" number along the mental number line. In Experiment 1, participants made temporal order judgements between digits presented to the left or to the right. The point of subjective simultaneity was shifted so that the 9 had to be presented before the 2 in order for simultaneity to be perceived. This difference could reflect either faster processing of the 2 or a response bias. Experiments $2 \mathrm{a}$ and $2 \mathrm{~b}$ eliminated response biases by using simultaneity judgements, which have no logical stimulus mapping. Both of the latter experiments established that the 2 was not processed faster than the 9 . Although the present results relate specifically to numerical magnitude and temporal order associations, they also have broader implications. Other studies have reported associations between dimensions such as size, duration, and number and have
\end{abstract}

\section{E. R. Nicholls $(\bowtie)$}

Brain \& Cognition Laboratory,

School of Psychology, Flinders University,

GPO Box 2100, Adelaide, South Australia 5001, Australia

e-mail: mike.nicholls@flinders.edu.au

T. Loetscher

School of Psychology, Flinders University,

GPO Box 2100, Adelaide, South Australia 5001, Australia

M. Lew $\cdot$ M. J. Yates

Department of Psychology, University of Melbourne,

Melbourne, Victoria, Australia attributed these to parietal mechanisms. Such associations, however, may also be an artefact of response biases.

Keywords ATOM - Mental number line - SNARC . Attention

Although dimensions such as time, number, and space have a largely distinct set of properties in the physical world, the manner in which the brain represents these dimensions is much less distinct. Associations in the cognitive and neural processing between these dimensions have been highlighted by Walsh (2003), who developed A Theory of Magnitude (ATOM), which brings the cortical representations of number, time, and space within a single explanatory framework (see also Bueti \& Walsh, 2009). Because the representation of magnitude is carried out by a common set of cognitive and neural mechanisms, shifts in one dimension are thought to induce a corresponding shift in the other dimensions.

In line with the ATOM theory, a link has been observed between the representations of numerical magnitude and space (Dehaene, 1997). In general, this research has demonstrated that smaller numbers are associated with the left side of space, whereas larger numbers are associated with the right. This "mental number line" has been demonstrated by requiring participants to make speeded parity (odd/even) judgements for small $(1,2)$ and large $(8,9)$ numbers. Responses are made with the left and right hands on buttons in the corresponding hemispace. Even though the magnitude of the numbers is irrelevant to the task, the left and right hands respond faster to numbers that are numerically smaller or larger, respectively (Dehaene, Bossini, \& Giraux, 1993). The association between numerical magnitude and space is known as the spatial numerical association of response codes (SNARC) effect (Dehaene et al., 1993). Some have suggested that the congruency effect between the left 
and right sides of space and numerical magnitude is related to response-related congruency effects, such as the Simon effect (Gevers, Lammertyn, Notebaert, Verguts, \& Fias, 2006; Keus \& Schwarz, 2005). Other researchers, however, have demonstrated that the link between number and space persists when there is no lateralised response (Casarotti, Michielin, Zorzi, \& Umiltà, 2007) or when the response dimension is orthogonal to the left/right dimension (Nicholls, Loftus, \& Gevers, 2008). A candidate site for the interaction between number and space is the right posterior parietal region, which codes the left and right sides of physical space (Bjoertomt, Cowey, \& Walsh, 2002) and is associated with the ability to shift attention along the mental number line (Göbel, Calabria, Farnè, \& Rossetti, 2006). It has been suggested that neural activity associated with shifts along one dimension produces a corresponding shift along the other dimension (Hubbard, Piazza, Pinel, \& Dehaene, 2005).

An association has also been observed between time and space. Vicario, Caltagirone, and Oliveri (2007) asked healthy participants to make time comparison judgements for periods lasting more and less than a second. Prior to making the judgements, participants were exposed to leftward or rightward optokinetic stimulation. The results revealed that exposure to leftward or rightward movement decreased or increased duration judgements, respectively. In a similar vein, Frassinetti, Magnani, and Oliveri (2009) used prismatic glasses to produce visuomotor shifts to the left or the right. Prismatic adaptation to the right produced an overestimation of time intervals, whereas adaptation to the left caused time intervals to be underestimated. Both studies appear to demonstrate that the left side of space is associated with "shorter" periods of time, whereas the right is associated with "longer" periods.

In addition to associations between number and space and time and space, there may also be a direct link between number and time. For temporal duration judgements, Oliveri et al. (2008) demonstrated that durations in which a relatively large digit (e.g., 9) was presented were judged to last longer than those in which a smaller digit (e.g., 1) was presented. A link between number and time has also been observed for temporal order judgements by Schwarz and Eiselt (2009). In their first experiment, digits were presented to the left and right of a central fixation point. The digits differed in their numerical magnitude and were either 3 and 4 (small numerical distance) or 1 and 9 (large numerical distance). The onset of the digits varied by an SOA of either 20 or $40 \mathrm{~ms}$. Numerical magnitude was irrelevant to the task, and participants were simply asked to respond "left first" or "right first." For digits separated by a large numerical distance, there was a clear effect of numerical magnitude. Trials on which the 1 preceded the 9 had a higher $d^{\prime}$ value than those with the reverse order, suggesting that the temporal structure of trials in an ascending order was easier to discriminate. No effect of numerical magnitude was observed for the small numerical distance. In a series of subsequent experiments, an association between magnitude and temporal order was observed across a wider range of SOAs for numbers, alphabetical stimuli, and combinations of letters and numbers.

Schwarz and Eiselt (2009) demonstrated that fewer errors are made for temporal order judgements when the stimuli are presented in their natural, left-to-right order (i.e., 1 presented before 9) To explain this association, they suggested that stimuli that occur earlier in a sequence (e.g., 1) have a shorter transfer latency to a central onset comparison stage. Thus, if iconic memory is read out in a left-to-right order, 1 will be processed earlier-leading to an easier temporal order judgement when the stimuli are presented in their natural sequence. When the stimuli are presented in the reverse order (e.g., 9 1), faster processing of the 1 will reduce the temporal interval between the stimuli, making the discrimination more difficult. Schwarz and Eiselt therefore believed that the association was driven by a mechanism related to the representation and processing of the stimulus.

Another possibility considered by Schwarz and Eiselt (2009) was based on a response bias mechanism. In this case, for trials on which participants were not sure which response to make, they would be more likely to choose the side on which the 1 occurred. The digit 1 occurs "earlier" in the left-to-right sequence, and this may map onto "which came first" for the temporal order judgement. The potential importance of linguistic factors such as this should not be underestimated. Gevers et al. (2010) demonstrated that the verbal coding of the response played an important role in the SNARC effect. By labelling responses as "left" or "right" and then varying the position of these responses, they demonstrated that the label was more important than the actual spatial location of the response. It is therefore possible that a similar response labelling exists for the task used by Schwarz and Eiselt (2009) and that this could explain their effect.

While Schwarz and Eiselt (2009) acknowledged that their results could be the product of a response bias, they argued that this was not the case, for a number of reasons. Three of these reasons were derived from manipulations of the stimulus properties of the temporal order task or by modelling the data (see Schwarz \& Eiselt, 2009, p. 1002, for a detailed discussion). In all three cases, some evidence was found for a response bias as well as for a genuine small-digit-first advantage. In addition, the effect of response biases was investigated by changing the nature of the response. In this case, participants completed a twoalternative forced choice task, which required them to discriminate between simultaneous versus successive trials (Exp.3). In this study, the digits 1 and 9 were presented so that either their onsets were simultaneous or one preceded the other by $10 \mathrm{~ms}$. Because responses of "simultaneous" 
and "successive" were orthogonal to the dimension of interest (small or large number), there could be no reason to suspect that the results would be influenced by a response bias. The results showed that " 1 -first trials enjoyed a $5 \%$ benefit as compared with performance on the 9-first trials" (Schwarz \& Eiselt, 2009, p.998). These results were taken to confirm that the association between numerical magnitude and temporal order reflected a genuine lower-number temporal processing advantage.

A few issues need to be taken into account when considering the impact of the simultaneity judgement task carried out by Schwarz and Eiselt (2009). First is the strength of the effects: For the temporal order judgement tasks, a clear and highly significant advantage was observed when the lower number preceded the higher number. For example, in Experiment 1, participants had an accuracy score of .83 in the 1-first trials, which dropped to .63 for the 9-first trials. The simultaneity judgement task, in contrast, showed an effect that only just reached statistical significance. In this case, the accuracy score for the 1-first trial was .67 , which dropped to .62 for the 9-first trials. Response bias therefore clearly played a dominant role in the association between numbers and temporal order. Second is the analysis, which appeared to be limited to the probability of correct responses for successive trials. Another way of analysing these data would be to examine the proportions of "simultaneous" and "successive" responses for both simultaneous and successive trials. If the 1-first processing advantage was genuine, it should also affect simultaneous trials by making them appear as if they were successive.

The potential impact of response bias on the link between numerical magnitude and temporal order is mirrored in other fields. For example, Schneider and Komlos (2008) were interested in reports by Carrasco, Ling, and Read (2004) that spatial cues affect the basic perceptual qualities of a stimulus, such as perceived contrast. Carrasco et al. presented spatial cues prior to the appearance of two Gabor gratings, and participants determined which of the targets had the higher contrast. The researchers found that cued targets were judged to have a higher perceived contrast. Schneider and Komlos replicated the effect observed by Carrasco et al. by asking participants to determine which of the stimuli had the higher contrast (comparative judgement). Another condition was introduced, however, in which participants judged whether or not the contrasts of the targets were equal (equality judgement). For equality judgements, no difference was observed between the cued and uncued targets. To explain the difference between the comparative and equality judgements, Schneider and Komlos argued that the comparative judgement used by Carrasco et al. was prone to a decision bias, which caused the cued target to be assigned a higher priority than the uncued target. Thus, participants decided that the cued target had a higher contrast because of an attentional biasing of the decision process, not because the target actually looked as if it had a higher contrast. The equality judgements were not prone to the same bias, because the response ("same"/"different") was not tied to a single target.

The research by Schneider and Komlos (2008) has important implications for studies purporting to demonstrate that shifts of spatial attention affect the appearance of a target. In a similar vein, it is also important to demonstrate unequivocally that numerical magnitude can affect the perceived timing of a stimulus, independent of decisional mechanisms. If a number's magnitude affected the way the timing of that stimulus was registered and processed, this would provide a significant new dimension to the ATOM model proposed by Walsh (2003). It would also demonstrate that relatively high-order mechanisms, such as numerical processing, can affect the temporal registration of a stimulus. However, if the association between numerical magnitude and timing proved to be the result of a response bias related to a linguistic/semantic association between different types of "first," it would be much less interesting. To examine this issue thoroughly, we have adopted a principled psychophysical analysis for temporal order and simultaneity judgement tasks.

\section{Experiment 1}

Experiment 1 was designed to replicate the effect of numerical processing on temporal order judgements observed by Schwarz and Eiselt (2009). The digits 2 and 9 were presented to the left and right of a central fixation point. The onset asynchrony between the digits was varied between 0 and $60 \mathrm{~ms}$, and participants indicated whether the left or right digit appeared first. Performance was measured by using a curve-fitting procedure to estimate the point of subjective simultaneity (PSS). It was expected that the point of subjective equivalence would be shifted towards 9 -first trials - indicating that the 9 needed to precede the 2 by some period of time for them to be perceived as simultaneous. This effect could be the result of two mechanisms. If the shift is the result of a genuine 2-first processing advantage, it would indicate that the 2 was transmitted earlier from short-term memory. Alternatively, it could be the result of a response bias, whereby participants conflate the response of "which first?" with the number that comes first along the mental number line (i.e., 2).

As in Schwarz and Eiselt (2009), the spatial congruency of the stimuli was also considered. It is possible that stimulus presentations on which the 2 falls on the left and the 9 on the right will be congruent with a left-to-right representation, resulting in an easier discrimination. In 
contrast, the opposite spatial configuration (i.e., 9 2) may make the temporal order discrimination more difficult. Schwarz and Eiselt observed no effect of spatial congruency in their experiments.

\section{Method}

Participants A total of 14 first-year psychology students (12 female, 2 male) participated as part of their course requirement. All were right-handed $(M=85.7, S D=17.0)$ as determined by the Edinburgh Handedness Inventory (Oldfield, 1971). Participants were between 18 and 23 years of age $(M=19.7, S D=1.6)$, and all had normal or corrected-to-normal vision and were naïve as to the purpose of the experiment, although prior informed consent had been obtained. The study had approval from the Melbourne University Human Research Ethics Committee.

Apparatus and stimuli Stimulus presentation was controlled via a PC with a digital input/output card and an onboard millisecond timer (Blue Chip Technology, DCM-16). Presentations were controlled using Turbo Pascal run in MS DOS. Stimuli were presented against a black display panel and viewed at a distance of $500 \mathrm{~mm}$. A central red LED $\left(0.2^{\circ}\right.$ wide) was placed in the centre of the panel. Two LED arrays (red, seven-segment numeric LED displays; part FND507) were placed so that their inner edges were $2.3^{\circ}$ of visual angle to the left or right of the central LED. The lateral LED arrays were $1.5^{\circ}$ high and $1.3^{\circ}$ wide. These arrays could display numbers between 0 and 9 with millisecond accuracy. A height-adjustable chinrest maintained participants' head position so that the centre of the display panel was in line with their midsagittal plane at eye level. A two-button response panel, which also lay in parallel with participant's midsagittal plane, was used to record responses. A closed-circuit video camera ensured that participants' concentration was maintained during the experiment. Participants who appeared to be moving their head or eyes too much were reminded to keep still and concentrate on the task.
Procedure See Fig. 1 for a sample trial sequence. Each trial began with illumination of the central LED, and participants were asked to direct their gazes towards this point. The central LED remained on throughout the trial. After $800 \mathrm{~ms}$ had elapsed, one of the lateral LED arrays was activated. Following a period lasting $0,5,10,15,30$, or $60 \mathrm{~ms}$, the other LED array was activated. Pretesting indicated that this range of SOAs would deliver levels of performance ranging from floor to ceiling, with many intermediate points. All three LEDs remained illuminated until a response was made by the participant. On one half of trials, the left LED array was illuminated first, and on the other half, the right was illuminated first. Half of the trials began with presentation of the 2 , and the other half began with the 9 . Although previous studies had used 1 and 9 to induce number and time congruencies (Schwarz \& Eiselt, 2009), the present study used 2 and 9 because they both require the illumination of five elements within the LED array, and therefore control for overall brightness.

Participants completed 528 trials, broken into six blocks. The factors of Side (left first, right first), SOA (six levels), and Number (2 first, 9 first) were equally represented within each block. The order in which the different factorial combinations were presented was randomised for each participant. Participants were asked to indicate which side was illuminated first as accurately as possible. Although RT was not stressed, trials with RTs longer than 4,000 ms were rejected and replaced by a trial with an identical configuration. This means that there were always 528 trials for analysis, but that some participants completed slightly more than this. The number of additional trials was small, and if participants were too slow to make a response, they were encouraged to respond more quickly. Participants were informed that the magnitude of the numbers was irrelevant to the task. Responses were made on a two-button response panel where the left and right buttons were used to indicate "left first" and "right first" responses. Because of the natural mapping between stimulus and response, the assignment of responses to buttons was not changed
Fig. 1 Representation of the stimulus presentation procedure used in Experiment 1. The figure shows spatially incongruent and congruent trials on which the left stimulus preceded the right. For the other half of the trials, the right stimulus preceded the left

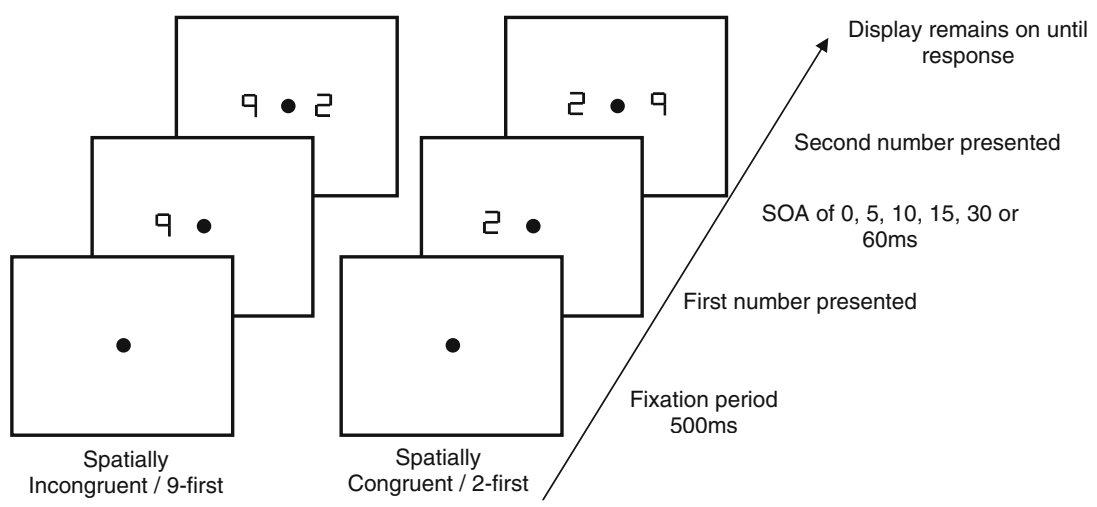


throughout the experiment. Following a response, a new trial began after 1,500 ms. Prior to the experimental trials, participants received 44 practice trials.

\section{Results and discussion}

The frequency of "2-first" responses was plotted as a function of SOA and fitted with a cumulative normal distribution function for each participant. Inspection of the plots for the participants suggested a good to very good fit, confirmed by corresponding $R^{2}$ values ranging from .65 to .98 , with a mean of .88. The PSS for each participant was extracted from these fitted functions by estimating the SOA value at which the curve crossed .5 on the $y$-axis (i.e., the point at which 9-first and 2-first responses were reported equally often). PSS values ranged from $-51.93 \mathrm{~ms}$ to $9.03 \mathrm{~ms}$, with a mean value of $-10.54 \mathrm{~ms}(S E=4.68)$. Negative PSS values indicated that the 9 had to be presented before the 2 in order for the digits to be perceived as simultaneous (and vice versa, for positive PSS values). The fitted function for the average results of each data point is shown in Fig. 2. A onesample $t$ test revealed a significant deviation of PSS values from true simultaneity towards the 9-first presentation condition $[t(13)=2.25, p=.041, d=0.60]$.

To investigate the effect of spatial congruency, the data were separated into spatially congruent (i.e., 2 9) and

\section{Experiment 1}

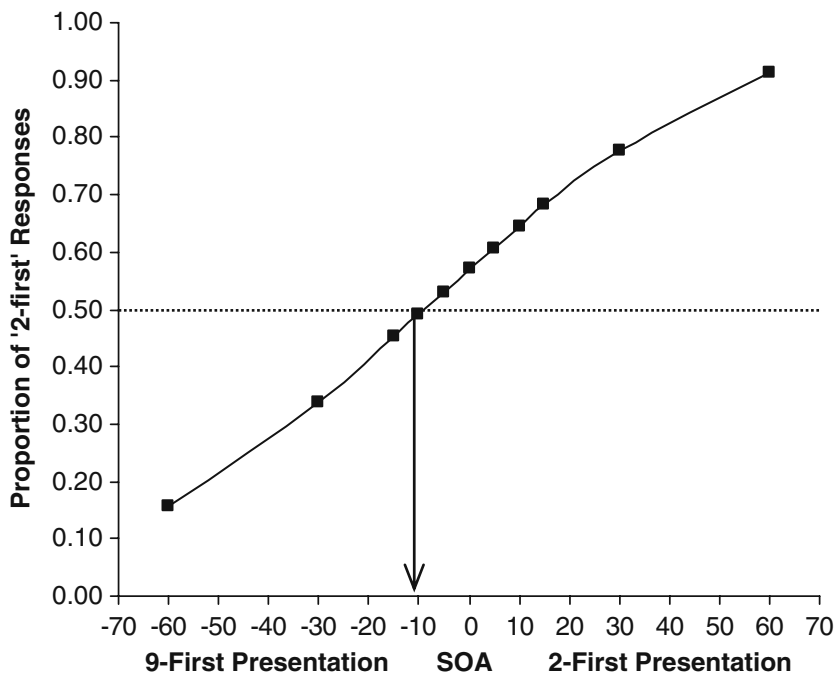

Fig. 2 Graph showing the proportions of "2-first" responses plotted against the SOA (in milliseconds) at which the 2 or 9 preceded the other stimulus in Experiment 1. Along the $x$-axis, shifts towards negative values indicate that the 9 needed to precede the 2 in order for them to be perceived as simultaneous (and vice versa, for positive values). The solid line is the best-fitting cumulative normal distribution, averaged across participants. The arrow shows the average point of subjective simultaneity spatially incongruent (i.e., 9 2) categories. As before, the data were fitted with a cumulative normal distribution, and the PSS was estimated. The mean PSS for the congruent condition $(M=-15.68 \mathrm{~ms}, S E=6.52)$ was not statistically different from the mean for the incongruent condition $(M=-4.90 \mathrm{~ms}, S E=6.46)[t(13)=1.22, p=.243]$. As in Schwarz and Eiselt (2009), therefore, it appears that the spatial configuration of the stimuli does not affect the perception of their temporal order.

In line with Schwarz and Eiselt (2009), the data suggest that numerical magnitude affects temporal order judgements. For the stimuli to be perceived as simultaneous, the 9 had to lead the 2 by $10.5 \mathrm{~ms}$. This result can be interpreted in two ways. The 10.5-ms advantage could reflect the operation of a mechanism related to the encoding and processing of the stimulus. In terms of the model put forward by Schwarz and Eiselt, this could mean that the 2 was transferred to a central onset comparison stage $10.5 \mathrm{~ms}$ more quickly than the 9 . The 10.5-ms advantage could also reflect a response bias. In cases where the participants were not sure of which response to make, they may have chosen the 2 , which has a natural association with the concepts "earlier" and "first." The increased frequency of 2-first responses would then produce a shift in the curve identical to the one shown in Fig. 2.

\section{Experiment 2a}

Experiment 1 replicated the effect of numerical magnitude on temporal order judgements observed by Schwarz and Eiselt (2009). As discussed above, the 10.5-ms advantage for 2-first trials could reflect the operation of either an encoding/processing mechanism or a response bias. Experiment 2a was designed to eliminate the effect of response biases. Like Schwarz and Eiselt, we used a simultaneity judgement task. Because judgements of "simultaneous" and "successive" are orthogonal to the dimensions of interest (i.e., first/last, earlier/later), there should be no response-mapping effect that could cause a response bias. The methodology of the present study differed in a number of ways from the one used by Schwarz and Eiselt. Rather than presenting two stimuli on each trial and requiring a two-alternative forced choice response for one or the other, we presented a single stimulus and required a two-alternative forced choice categorisation. The advantage of this technique is that the stimulus presentation procedure was identical to the one used in Experiment 1-except for the response. This correspondence would allow us to draw stronger inferences from any differences between the experiments. Schwarz and Eiselt appear to have limited their analysis to the proportions correct for the successive trials only. This experiment could instead use all of the data to generate curves to estimate the PSS. 
If there is a genuine temporal processing advantage for lower digits, there should be a shift in the PSS towards 9-first trials. That is, because the 2 is processed more quickly than the 9 , the 9 would need to lead the 2 by some period of time in order for them to be perceived as simultaneous. If no shift occurs, it would suggest that there is no difference in processing speed between small and large digits and that the effects observed for temporal order tasks are the result of a response bias.

\section{Method}

Participants A total of 14 first-year psychology students (10 female, 4 male) participated in the experiment. All were right-handed $(M=82.8, S D=13.2)$ and were between 18 and 42 years of age $(M=22.4$ years, $S D=7.3)$. All other characteristics were the same as in Experiment 1.

Stimuli and procedure The methodology was the same as in Experiment 1, except for one change. Instead of indicating which stimulus came first, participants indicated whether the onset of the stimuli was simultaneous or successive. Participants pushed the left or the right button to indicate that the stimuli were simultaneous or successive, and the laterality of these responses was reversed between participants. The participants were given no information about the relative proportions of simultaneous and successive trials.

\section{Results and discussion}

The frequency of simultaneous responses was plotted as a function of SOA and fitted with a Gaussian distribution function for each participant. Inspection of the plots for the participants suggested a good to very good fit, confirmed by corresponding $R^{2}$ values ranging from .55 to .98 , with a mean of .89. Although the value of .55 is quite low, we decided to keep the data from participants with such low values to maintain the representativeness of our sample. The PSS for each participant was extracted from these fitted functions by estimating the SOA value at the point where simultaneous responses were maximal. In a Gaussian distribution, this point is $\mu$ (Bolker, 2008), and therefore the PSS value was derived from the mean value generated by the nonlinear regression. The PSS values ranged from $-1.72 \mathrm{~ms}$ to $0.48 \mathrm{~ms}$, with a mean value of $-0.30 \mathrm{~ms}(S E=0.21)$. The fitted function for the average results for each data point is shown in Fig. 3. As can be seen in the figure, the obtained PSS did not differ significantly from zero $[t(13)=1.46, p=.173]$, indicating that participants did not show a systematic tendency to perceive the numbers as simultaneous at any other point

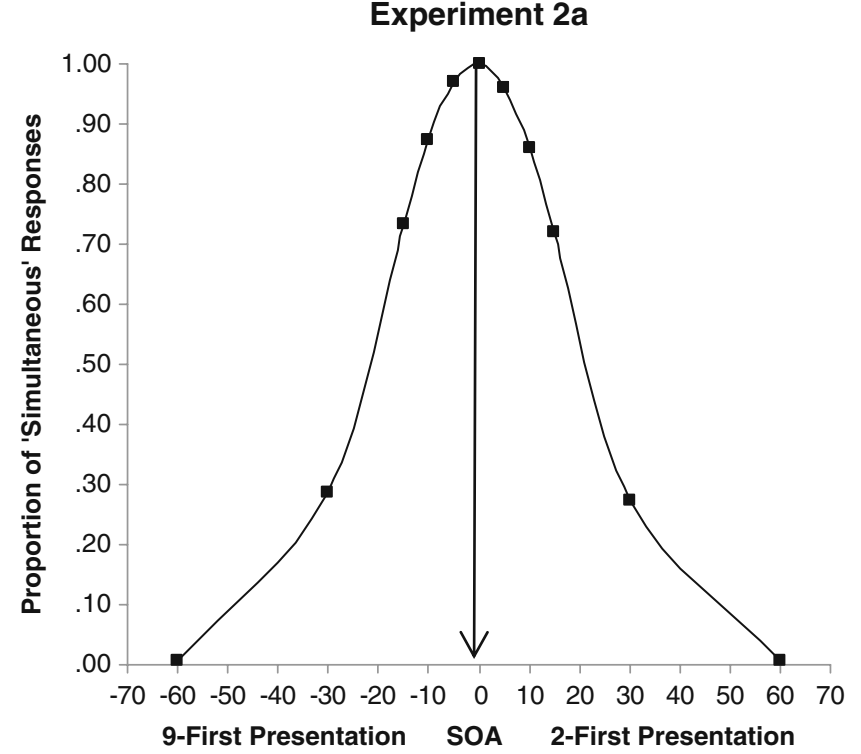

Fig. 3 Graph showing the proportions of "simultaneous" responses plotted against the SOA (in milliseconds) at which the 2 or 9 preceded the other stimulus in Experiment 2a. The solid line is the best-fitting Gaussian function, averaged across participants. The arrow shows the average point of subjective simultaneity

other than when the presentation truly was simultaneous. Examination of the spatially congruent $(M=-2.67 \mathrm{~ms}, S E=$ $1.74)$ and incongruent $(M=-0.43 \mathrm{~ms}, S E=0.26)$ conditions revealed no statistically significant difference between them $[t(13)<1]$, verifying that spatial configuration did not influence participants' simultaneity judgements.

The stimuli used in this experiment were identical to those used in Experiment 1. Despite this, no 2-first advantage was observed. If there were a genuine 2 -first processing advantage, the curve should have been shifted to the left, showing that the 9 needed to precede the 2 by some period of time to be perceived as simultaneous. Given that the nature of the decision and response is the only thing that changed from Experiment 1, it seems likely that this dimension is crucial in generating the lower-digit advantage observed by Schwarz and Eiselt (2009).

\section{Experiment 2b}

It is problematic to provide support for a theory by showing a null effect. For example, the failure to find a difference in processing speed between the digits in Experiment $2 \mathrm{a}$ could be the result of a lack of statistical power (not enough trials or participants) or of an experimental design that did not capture the relevant behaviour. With this in mind, Experiment $2 \mathrm{~b}$ was designed to increase the chances of observing a 2-first advantage for simultaneity judgements. Inspection of Fig. 3 reveals that 
$64 \%$ of the SOAs are associated with a .70 or higher probability of responding "simultaneous." It is possible that the concentration of data towards the ceiling of simultaneous responses may have militated against a significant effect. To avoid this, we spread the SOAs so that they yielded data from a larger section of the curve. Experiment 1 produced an effect using just 14 participants. Although it seems reasonable to assume that the simultaneity judgements should produce an effect using a similar number of participants, in the present experiment we increased their number to 26 to increase the statistical power of the test.

\section{Method}

Participants A total of 26 first-year psychology students (21 female, 5 male) participated in the experiment. All were right-handed $(M=83.4, S D=23.6)$ and were between 18 and 21 years of age $(M=19.6$ years, $S D=$ 1.1). All other characteristics were the same as in Experiment 1.

Stimuli and procedure The methodology was the same as in Experiment 2a, except that the SOAs were changed to 0, $15,25,36$, and $60 \mathrm{~ms}$.

\section{Results and discussion}

The data were fitted and analysed using the procedure described for Experiment 2a. Inspection of the plots for the participants suggested a good to very good fit, confirmed by corresponding $R^{2}$ values ranging from .47 to .98 , with a mean of .90 . The PSS values ranged from -3.12 to $2.05 \mathrm{~ms}$, with a mean value of $-0.27 \mathrm{~ms}(S E=0.22)$. The fitted function for the average results for each data point is shown in Fig. 4. As can be seen in the figure, the obtained PSS did not differ significantly from zero $[t(25)=1.26$, $p=.224]$, indicating that participants did not show a systematic tendency to perceive the numbers as simultaneous at any point other than when the presentation truly was simultaneous. Examination of the spatially congruent $(M=0.74 \mathrm{~ms}, S E=0.56)$ and incongruent $(M=1.01 \mathrm{~ms}$, $S E=0.65)$ conditions revealed no statistically significant difference between them $[t(25)=1.61, p=.120]$, verifying that spatial configuration did not influence participants' simultaneity task performance.

The results are effectively identical to those observed in Experiment 2a. Despite spreading the range of SOAs and increasing the number of participants, the point of subjective simultaneity did not differ from true simultaneity. Experiment $2 \mathrm{~b}$ therefore adds further weight to the proposition that the lower-digit temporal processing advan-

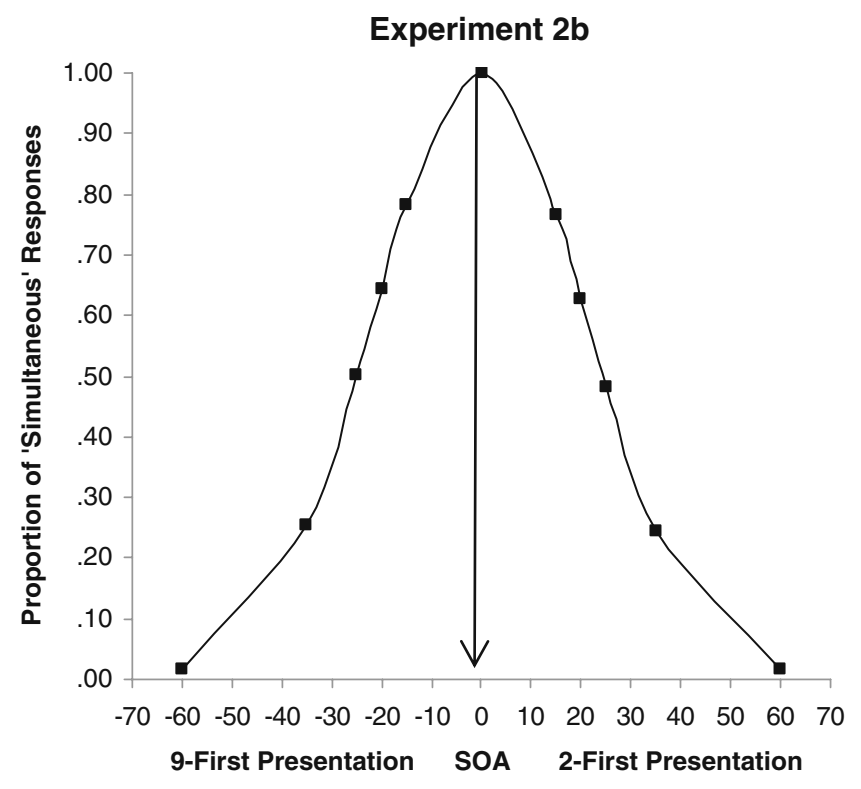

Fig. 4 Graph showing the proportions of "simultaneous" responses plotted against the SOA (in milliseconds) at which the 2 or 9 preceded the other stimulus in Experiment 2b. The solid line is the best-fitting Gaussian function, averaged across participants. The arrow shows the average point of subjective simultaneity

tage is the result of a response bias rather than a genuine processing advantage.

\section{General discussion}

Experiment 1 replicated the association between temporal order judgements and numerical magnitude observed by Schwarz and Eiselt (2009). Like them, we observed a robust effect whereby the 9 had to precede the 2 in order for the two numbers to be perceived as simultaneous. On balance, Schwarz and Eiselt believed that this association was the result of a processing advantage for smaller digits, which caused the lower digit to be read out earlier from short-term memory. It is also possible, however, that the association was the result of a response bias. Experiments $2 \mathrm{a}$ and $2 \mathrm{~b}$ eliminated the effects of response biases by using a simultaneity judgement task. Responses of "simultaneous" and "successive" have little logical connection with numerical magnitude, and therefore should not be prone to the linguistic/semantic associations that cause response biases. It could be argued that a within-subjects design, such as the one used by Schneider and Komlos (2008), would provide a more powerful test of differences between the conditions. However, this sort of design has the pitfall of contamination between the conditions, even if their order is controlled between subjects. In addition, care was taken to select our participants from the same pool, with the same age range and roughly the same sex ratio, which should 
have significantly reduced any between-group differences. It might also be argued that "simultaneous" is coded as smaller than "successive," and therefore that "simultaneous" may lie to the left of "successive" along a mental number or magnitude line. If this were the case, however, the PSS should have been shifted to the left, as predicted by the general temporal/numerical processing model. The results of Experiments 2a and 2b convincingly demonstrated no shift of subjective simultaneity away from the point of true simultaneity. The association between temporal order judgements and numerical magnitude therefore appears to be dependent on the response and not to be the result of a temporal processing advantage for smaller digits.

Although the temporal order and simultaneity tasks have been used interchangeably in the present study to measure temporal sensitivity, it is important to consider the properties of the tasks and whether they depend on a common set of neural and cognitive mechanisms. Brain research has pointed to a similar set of neural mechanisms. Battelli, PascualLeone, and Cavanagh (2007) suggested that the temporal processing of events at an intermediate time scale, such as temporal order and simultaneity judgements, is carried out in a common "when" centre located in the right inferior parietal lobe. Nicholls (1996) also suggested that temporal order and simultaneity tasks are carried out in the same region-but in this case, the left temporal lobe was the candidate region.

With respect to the cognitive mechanisms that underlie temporal order and simultaneity judgements, the evidence is mixed (Vatakis, Navarra, Soto-Faraco, \& Spence, 2008). Some researchers have found that temporal order and simultaneity judgement tasks yield similar levels of performance (e.g., Baron, 1969; Vroomen, Keetels, de Gelder, \& Bertelson, 2004). In contrast, Mitrani, Shekerdjiiski, and Yakimoff (1986) found that temporal order judgements could not be made, despite that fact that the stimuli were perceived to be successive. Mitrani et al. therefore concluded that the two types of judgement were the product of independent mechanisms for evaluating temporal interrelations. Interestingly, and particularly relevant to the present study, Vatakis et al. (2008) suggested that the differences between the temporal order and simultaneity tasks were related to response biases, which can affect either task. For temporal order judgements, biases towards "which first" or "which last" can play an important role (Shore, Spence, \& Klein, 2001). Within the context of simultaneity judgements, an assumption that the stimuli are bound together within a spatial location (Shore, Spry, \& Spence, 2002) or within a modality (Vatakis \& Spence, 2007) can encourage participants to respond to the temporal properties of the stimuli as "simultaneous" more often than "successive." Within the present context, differential susceptibility to response biases also provides a good explanation of the results. Thus, both tasks tap the same temporal mechanism, which shows no difference in the speed of processing for small or large digits. However, temporal order judgements are prone to response biases, which increase the likelihood of "2-first" responses.

Although it seems reasonable to assume that temporal order and simultaneity judgements engage a similar set of neural and cognitive mechanisms related to temporal processing, it is still possible that participants approached the tasks with different "attentional sets" or strategies. These differences could account for the divergence between the temporal order and simultaneity tasks. At one level, it could be argued that these effects should be minimal. The display, stimuli, and response mechanism were identical between the tasks, and therefore should encourage very similar approaches. At another level, it could be acknowledged that strategy effects do exist, but do not affect the ultimate conclusion. That is, if there were a real link between numerical magnitude and temporal order perception, this link should be apparent for both temporal order and simultaneity tasks, regardless of whether a different strategy or attentional set was adopted.

Schneider and Komlos (2008) attempted to control for strategy differences by presenting stimuli and then informing participants whether they should make a comparative or equality judgement. By presenting the type of response after stimulus presentation, the attentional set and/or strategy should be consistent between tasks. Schneider and Komlos found that the difference between the alternative types of judgement was markedly reduced when they were intermixed within participants-though it was still significant. Interestingly, they also noted that participants found the intermixed trials difficult and that most reported "mainly preparing for the comparative judgement and switching to the equality judgement when necessary" (Schneider \& Komlos, 2008, p. 7). It is very likely that this strategy overcame many of the advantages of the intermixed design and accounted for the small difference between the judgement conditions. If an intermixed design had been adopted in the present study, it is likely that a similar result would have been observed. That is, despite not knowing what sort of judgement was required, most participants would prepare for one task and then switch if necessary. Although it is difficult to predict a priori what task would be preferred, Jaskowski (1991) reported that knowledge of simultaneity was needed before a temporal order judgement could be made. Therefore, it is possible that participants could have prepared for a simultaneity judgement and then switched if necessary.

The results of the present study are analogous to those of Schneider and Komlos (2008), who demonstrated that attention biased decisions but did not affect stimulus contrast. Schneider and Komlos considered the likely locus of the cue effect and proposed that it occurred at a level 
where attention induced an assignment of higher priority to the cued target-leading to a bias towards selecting that target. Although the present data also demonstrate that biased decisions affect a task, the locus of the effect is different. In this case, rather than attention biasing the decision, the bias here derives from a higher-order mechanism related to the conceptual coding of numerical magnitude and temporal order. The results are also consistent with studies showing that the nature of the response plays an important role in temporal processing studies (Shore et al., 2001). Despite the fact that findings such as these have been published, not all studies have taken response biases and decisional mechanisms into account. It is therefore important to demonstrate the role of response biases within an experimental paradigm. In the present case, we have demonstrated that numerical magnitude does not affect the temporal order of stimuli if response biases are controlled.

The findings of this study have important implications for the ATOM model proposed by Walsh (2003), which provides a structure for understanding the interrelationships between judgements of magnitude. Within this framework, links have been observed between numerical magnitude and space (Dehaene et al., 1993; Nicholls et al., 2008), time and space (Frassinetti et al., 2009; Vicario et al., 2007), and number and time (Oliveri et al., 2008). With all of these relationships, however, one needs to be careful about the impact of responses biases. That is, the relationship between different scales of magnitude may be related to the linguistic/semantic interrelationships between the stimulus and the response. The importance of verbal labels has recently been highlighted by Gevers et al. (2010). Besides the present example of Schwarz and Eiselt (2009), there may be other cases. For example, Xuan, Zhang, He, and Chen (2007) asked participants to make relative duration judgements (e.g., which one is shorter/longer?) for stimuli that varied in their presentation duration and physical magnitude. The results showed lower levels of error for congruent trials, on which the shorter period coincided with the stimulus with the lower physical magnitude. For example, a small square was judged to last for a shorter duration than a large square. Although Xuan et al. discussed the possibility that their results emerged because of a parietal mechanism related to the ATOM model (Walsh, 2003), it is also possible that their findings were the result of a response bias. That is, when participants were unsure of which response to make, they biased their responses towards the stimulus that naturally aligned with the response. Because "small" and "shorter" are naturally aligned, participants could have biased their responses towards the small stimulus when asked to determine which stimulus was shorter (and vice versa, when asked for the stimulus with longer duration). To avoid such associations, the response needs to be orthogonal to the stimulus dimension of interest. In the case of Xuan et al.'s study, participants could have made same-different judgements instead. By employing methods such as this, stronger evidence for links between the processing of different magnitudes would be obtained.

\section{References}

Baron, J. (1969). Temporal ROC curves and the psychological moment. Psychonomic Science, 15, 299-300.

Battelli, L., Pascual-Leone, A., \& Cavanagh, P. (2007). The "When" pathway of the right parietal lobe. Trends in Cognitive Sciences, $11,204-210$.

Bjoertomt, O., Cowey, A., \& Walsh, V. (2002). Spatial neglect in near and far space investigated by repetitive transcranial magnetic stimulation. Brain, 125, 2012-2022.

Bolker, R. B. M. (2008). Ecological models and data. Princeton: Princeton University Press.

Bueti, D., \& Walsh, V. (2009). The parietal cortex and the representation of time, space, number and other magnitudes. Philosophical Transactions of the Royal Society, 364, 1831-1840.

Carrasco, M., Ling, S., \& Read, S. (2004). Attention alters appearance. Nature Neuroscience, 7, 308-313.

Casarotti, M., Michielin, M., Zorzi, M., \& Umiltà, C. (2007). Temporal order judgements reveal how number magnitude affects visuospatial attention. Cognition, 102, 101-117.

Dehaene, S. (1997). The number sense: How the mind creates mathematics. New York: Oxford University Press.

Dehaene, S., Bossini, S., \& Giraux, P. (1993). The mental representation of parity and number magnitude. Journal of Experimental Psychology: General, 122, 371-396.

Frassinetti, F., Magnani, B., \& Oliveri, M. (2009). Prismatic lenses shift time perception. Psychological Science, 20, 949-954.

Gevers, W., Lammertyn, J., Notebaert, W., Verguts, T., \& Fias, W. (2006). Automatic response activation of implicit spatial information: Evidence from the SNARC effect. Acta Psychologica, 122, 221-233.

Gevers, W., Santens, S., Dhooge, E., Chen, Q., Van den Bossche, L., Fias, W., \& Verguts, T. (2010). Verbal-spatial and visuospatial coding of number-space interactions. Journal of Experimental Psychology: General, 139, 180-190.

Göbel, S. M., Calabria, M., Farnè, A., \& Rossetti, Y. (2006). Parietal rTMS distorts the mental number line: Simulating "spatial" neglect in healthy subjects. Neuropsychologia, 44, 860-868.

Hubbard, E. M., Piazza, M., Pinel, P., \& Dehaene, S. (2005). Interactions between number and space in parietal cortex. Nature Reviews. Neuroscience, 6, 435-448.

Jaskowski, P. (1991). Two-stage model for order discrimination. Perception \& Psychophysics, 50, 76-82.

Keus, I. M., \& Schwarz, W. (2005). Searching for the functional locus of the SNARC effect: Evidence for a response-related origin. Memory \& Cognition, 33, 681-695.

Mitrani, L., Shekerdjiiski, S., \& Yakimoff, N. (1986). Mechanisms and asymmetries in visual perception or simultaneity and temporal order. Biological Cybernetics, 54, 159-165.

Nicholls, M. E. R. (1996). Evidence and implications of a temporal processing dichotomy between the hemispheres. Laterality, 1, 97-137.

Nicholls, M. E. R., Loftus, A., \& Gevers, W. (2008). Look, no hands: A perceptual task shows that number magnitude induces shifts of attention. Psychonomic Bulletin \& Review, 15, 413-418. 
Oldfield, R. C. (1971). The assessment and analysis of handedness: The Edinburgh inventory. Neuropsychologia, 9, 97-113.

Oliveri, M., Vicario, C. M., Salerno, S., Koch, G., Turriziani, P., Mangano, R., et al. (2008). Perceiving numbers alters time perception. Neuroscience Letters, 438, 308-311.

Schneider, K. A., \& Komlos, M. (2008). Attention biases decisions but does not alter appearance. Journal of Vision, 8(15), 3:1-10.

Schwarz, W., \& Eiselt, A. (2009). The perception of temporal order along the mental number line. Journal of Experimental Psychology: Human Perception and Performance, 35, 989-1004.

Shore, D. I., Spence, C., \& Klein, R. M. (2001). Visual prior entry. Psychological Science, 12, 205-212.

Shore, D. I., Spry, E., \& Spence, C. (2002). Confusing the mind by crossing the hands. Cognitive Brain Research, 14, 153-163.

Vatakis, A., Navarra, J., Soto-Faraco, S., \& Spence, C. (2008). Audiovisual temporal adaptation of speech: Temporal order versus simultaneity judgements. Experimental Brain Research, 185, 521-529.

Vatakis, A., \& Spence, C. (2007). Crossmodal binding: Evaluating the "unity assumption" using audiovisual speech stimuli. Perception \& Psychophysics, 69, 744-756.

Vicario, C. M., Caltagirone, C., \& Oliveri, M. (2007). Optokinetic stimulation affects temporal estimation in healthy humans. Brain and Cognition, 64, 68-73.

Vroomen, J., Keetels, M., de Gelder, B., \& Bertelson, P. (2004). Recalibration of temporal order perception by exposure to audio-visual asynchrony. Cognitive Brain Research, 22, 3235.

Walsh, V. (2003). A theory of magnitude: Common cortical metrics of time, space and quantity. Trends in Cognitive Sciences, 7, 483-488.

Xuan, B., Zhang, D., He, S., \& Chen, X. (2007). Larger stimuli are judged to last longer. Journal of Vision, 7(10), 2:1-5. 